10.2478/v10367-012-0012-x

NAM-SUN SON, SUN-YONUG KIM, CHAN-SOO LEE

Kordi, Republic of Korea

\title{
ON THE MONITORING SYSTEM \\ OF COLLISION RISKS AMONG MULTIPLE SHIPS
}

\begin{abstract}
To reduce crewmembers' error and increase the safety of ships' navigation, a recognition system of collision risks among multiple ships is newly-developed. By using ships' navigational information such as AIS data, an algorithm to calculate collision risks among multiple ships is newly-designed. Collision risks of multiple ships can be estimated considering ships' position and course of now and future by using fuzzy algorithm. To evaluate the performance of new system, replay simulation is carried out by using actual AIS data of actual collision accident in Korea. In this paper, main features of the monitoring system of collision risks and the results of replay simulation are discussed.
\end{abstract}

Keywords:

collision risk among multiple ships.

\section{INTRODUCTION}

In spite of navigational support systems such as radar, ECDIS and AIS, ship collision accidents have not been decreased. According to the statistics of maritime accidents, about $30 \%$ of maritime accidents are colliding accidents and more than $90 \%$ of them are due to human error [1].

Actually, it takes time and procedure from initial recognition of dangerous traffic ship to collide with ownship to the action of collision avoidance. So, it is necessary to collect the navigational information on many traffic ships and analyze the mutual collision risk among multiple ships and recognize risky traffic ship to collide with ownship, automatically.

In order to quantify the collision risk, Imazu and Koyama defined a probability of collision, using the error characteristics of systems like radars [5]. The concept of blocking area also has been used for collision avoidance. Furukawa and Kijima studied on the automatic collision avoidance system using the concept of blocking area, 
which is used to evaluate the collision risk among ships [8]. Wilson and Harris assessed risk of collision by using the line-of-sight (LOS) which is the vector from target ship to own-ship [17]. Szlapczynski derived collision risk by using the concept of circle-shaped ship domain [15]. Kao calculated danger index by using the difference between the safety time interval and the discrepancy of collision point for target ships [7]. In most algorithms, collision risk is estimated by using a kind of time to the collision or a kind of distance to the collision.

To estimate the collision risk effectively, fuzzy algorithm also has been used. Hasegawa inferred the collision risk using time to the closest point of approach (TCPA) and distance to the closest point of approach (DCPA) [4]. Hara inferred the range of collision risk [3]. Lee extended the algorithm of collision avoidance on a basis of Hasegawa's results [10]. Kijima and Furukawa designed automatic collision avoidance system using fuzzy inference [9]. Son estimate the directional collision risk around own-ship by using fuzzy algorithm [11]. On the other hand, environmental stress, which crewmember feels in colliding situation, has been used for collision risk. Inoue analyzed the environmental stress on a basis of Hara's result that the environmental stress can be defined with the relative position and length average of own-ship and traffic ship [6]. Gong applies the environmental stress model due to geographical restriction, to the design of fairway in a harbor [2]. Son compared the collision risk calculated by using fuzzy algorithm, together with environmental stress [12]. Son also designed an algorithm to estimate the collision risk among ships and he applied the algorithm to the actual AIS data [13, 14].

To reduce crewmembers' error and help them recognize colliding situation quickly, an onboard-type Collision Risks Monitoring System (CRMS) is newly developed. Based on AIS data as traffic ships' navigational information, collision risks among multiple ships are estimated. Here, collision risks of multiple ships can be calculated considering ships' course of now and future by using fuzzy algorithm. To test the performance of CRMS, replay simulation is carried out by using AIS data of actual ships' collision accident in Korea. AIS data include 244 ships' information for two hours. In this paper, features of newly-developed CRMS and the results of replay simulation are discussed.

\section{DESIGN OF MONITORING SYSTEM OF COLLISION RISKS AMONG SHIPS}

To help navigational officer recognize very dangerous traffic ship on his ownship and take proper action quickly, a real-time collision risk monitoring system among ship (CRMS) is newly developed as an onboard system. 
The estimation procedure of CRMS is shown in fig. 1. It is assumed that traffic ships' navigational data are collected from AIS. By using fuzzy algorithm, collision risks between all combinations of ships are estimated among multiple ships including own-ship in real time [12]. According to the level of collision risk, different color circle is overlaid on each traffic ship in Electronic Navigation Chart (ENC). Directional collision risks of ownship are also calculated and displayed.

Fig. 2 shows the procedure to estimate collision risk by using fuzzy algorithm.

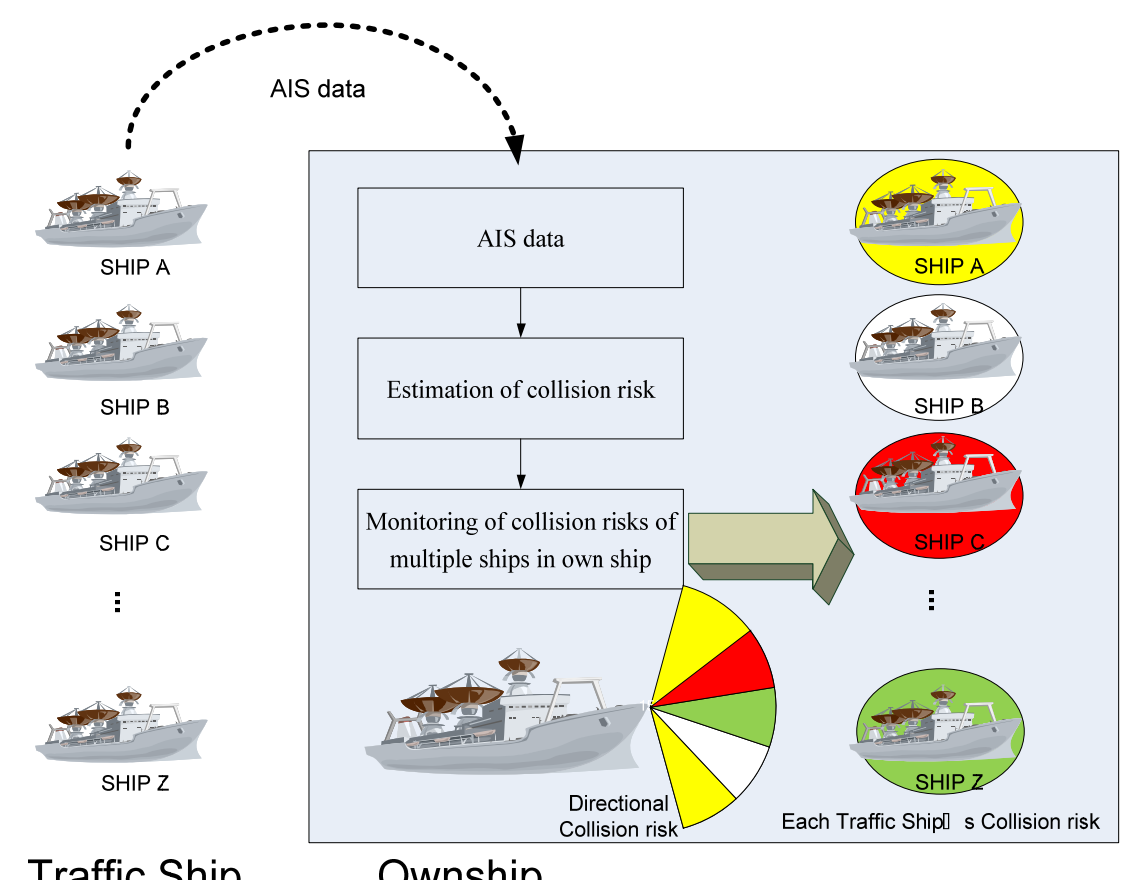

Fig. 1. Procedure of CRMS [own study]

Here, $i, j$ and $k$ mean the index of ship of which collision risk is calculating, the index of other traffic ship except for $i$-th ship and the index of $i$-th ship's direction which is assumed to calculate collision risk in future, respectively. $P_{i}$ means AIS data of $i$-th ship. $N_{T}$ and $N_{\theta}$ mean the number of all ships and the number of unit direction. In this paper, if $\mathrm{k}$ is zero, direction is $-75 \mathrm{deg}$ and unit direction is $30 \mathrm{deg}$. $N_{\theta}$ is set to 5 . 


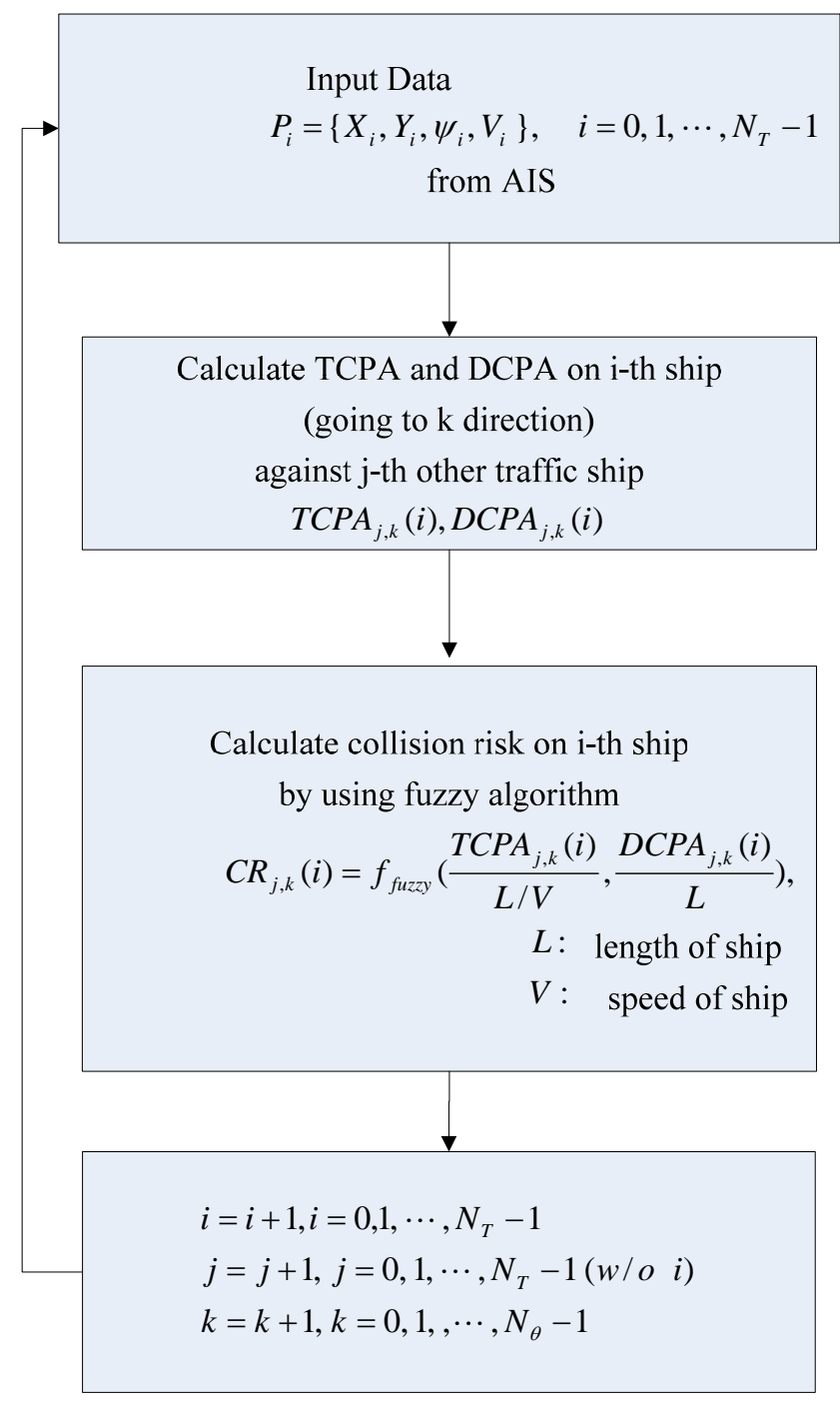

Fig. 2. Flow chart of estimation algorithm of collision risk among multiple ships [14]

As shown in fig. 2, collision risks among multiple ships are as follows [14]:

1. To calculate collision risk of $i$-th ship, assuming that $i$-th ship is going to $k$-th direction, $T C P A_{j, k}(i)$ and $D C P A_{j, k}(i)$ are calculated against $j$-th other ship.

2. Increasing $j$ index, $C R_{j, k}(i)$ is calculated on each $j$-th other ship by using fuzzy algorithm. The representative collision risk $C R_{k}(i)$ of $i$-th ship in $k$-th 
direction is decided as the maximum collision risk out of all collision risk on all other traffic ships as equation (1).

$$
C R_{k}(i)=\max \left(C R_{j, k}(i)\right) .
$$

3. Increasing $k$ index, $C R_{k}(i)$ is calculated on each $k$-th direction by conducting above (2) procedure repeatedly. The representative collision risk of $i$-th ship $C R(i)$ is calculated considering directional weighting factor $w(\theta)$ as equation (2).

$$
C R(i)=\frac{\int_{\theta_{0}}^{\theta_{f}} C R_{k}(i) \cdot w(\theta) d \theta}{\int_{\theta_{0}}^{\theta_{f}} 1 \cdot w(\theta) d \theta},
$$

where

$$
\theta=\psi_{i}+30 \cdot(k-2), w(\theta)=\cos \left(\frac{90}{110} \theta\right)
$$

4. Increasing $i$ index, $C R(i)$ of each $i$-th ship is calculated by conducting above (3) procedure repeatedly. $C R(i)$ has the range of -1.0 to 1.0 .

Therefore, we can find each collision risk of each ship. The color of ship and direction can be changed according to the level of collision risk. In case that $C R(i)$ is lower than 0.4 , ship is assumed to be safe. In case that $C R(i)$ is 0.4 to 0.6 , ship is assumed to be marginally safe. In case that $C R(i)$ is 0.6 to 0.8 , ship is assumed to need caution. In case that $C R(i)$ is more than 0.8, ship is assumed to be very dangerous. The level of directional collision risk, $C R_{k}(i)$ is that of $C R(i)$. So, most dangerous ship or ships to collide each other can be found according to the value of collision risk. The constant values of criteria need to be tuned through a number of validations based on AIS data and field tests.

\section{TESTBED OF MONITORING SYSTEM OF COLLISION RISKS AMONG SHIPS}

To inform navigational officer and coast pilot of collision risk for safe arrival and departure near coastal area, the test-bed of real-time collision risk monitoring system among ship (CRMS) is implemented as hand-carry onboard system. The main configuration of CRMS is shown in fig. 3 . 


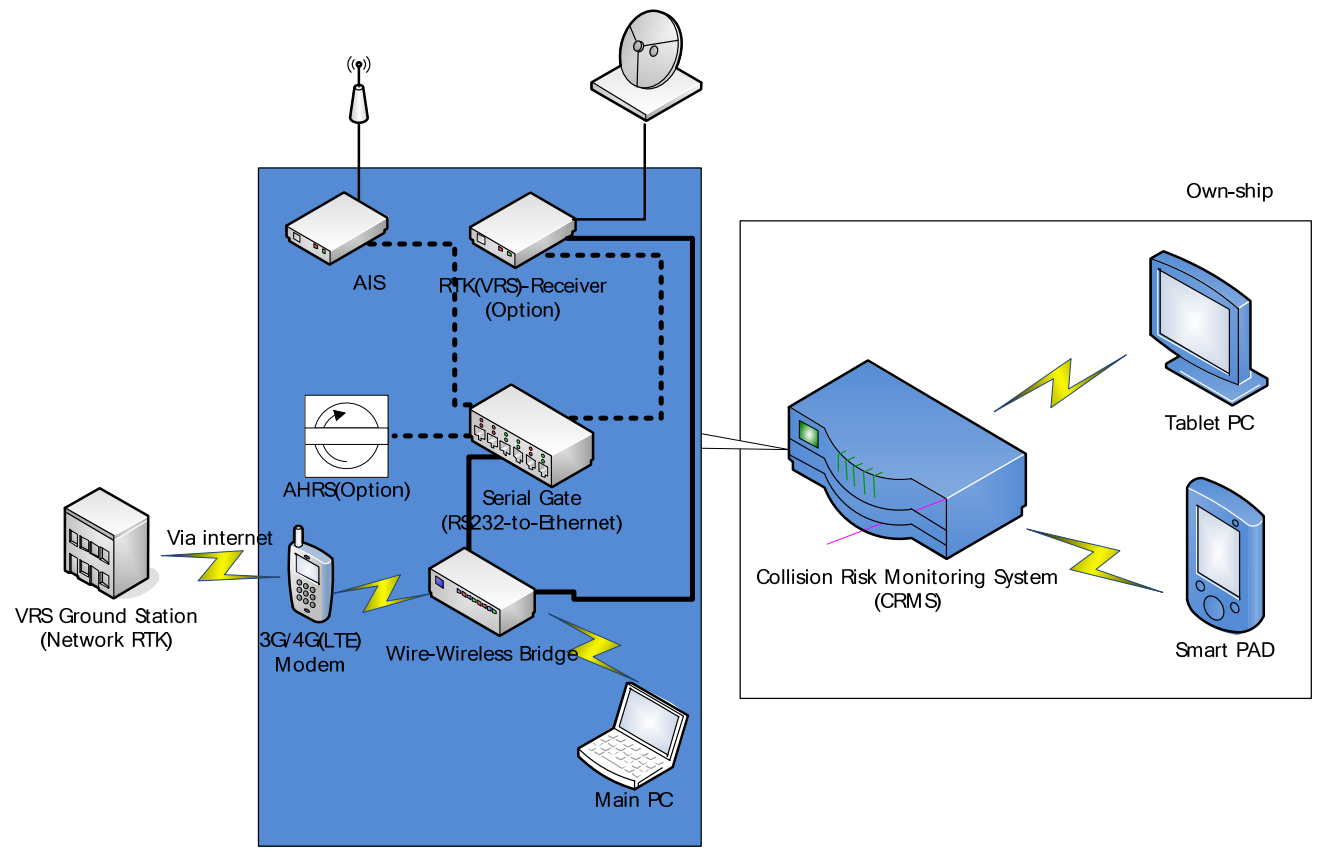

Fig. 3. Configuration of CRMS [own study]

For traffic information, AIS is enough but Real-Time Kinematic (RTK) GPS is installed for precise measurement of ownship's position during arrival and departure near coastal area. As a network RTK, Virtual Reference Station (VRS) service has been initiated in Korea since November, 2007. To receive the position correction data of VRS via internet, 3G/4G(LTE) modem is also installed. Attitude Heading Reference System (AHRS) is installed to acquire magnetic heading and rate of turn (ROT) of ownship. All navigational information of AIS, RTK-GPS and AHRS are collected in serial gate and transmitted to main PC via wireless bridge. Position, course of ground (COG) and speed of ground (SOG) of traffic ships are received from AIS. Position, COG and SOG of ownship are received from RTK-GPS. Magnetic heading, ROT and attitude such as roll and pitch are received from AHRS. Fig. 4 shows the fully-installed test-bed of CRMS. The test-bed box is sensor box and it can be used as docking station for main PC. The test-bed can be hand-carried by user like coast pilot. Graphic User Interface (GUI) based on IHO S-57 ENC is shown in fig. 5. 


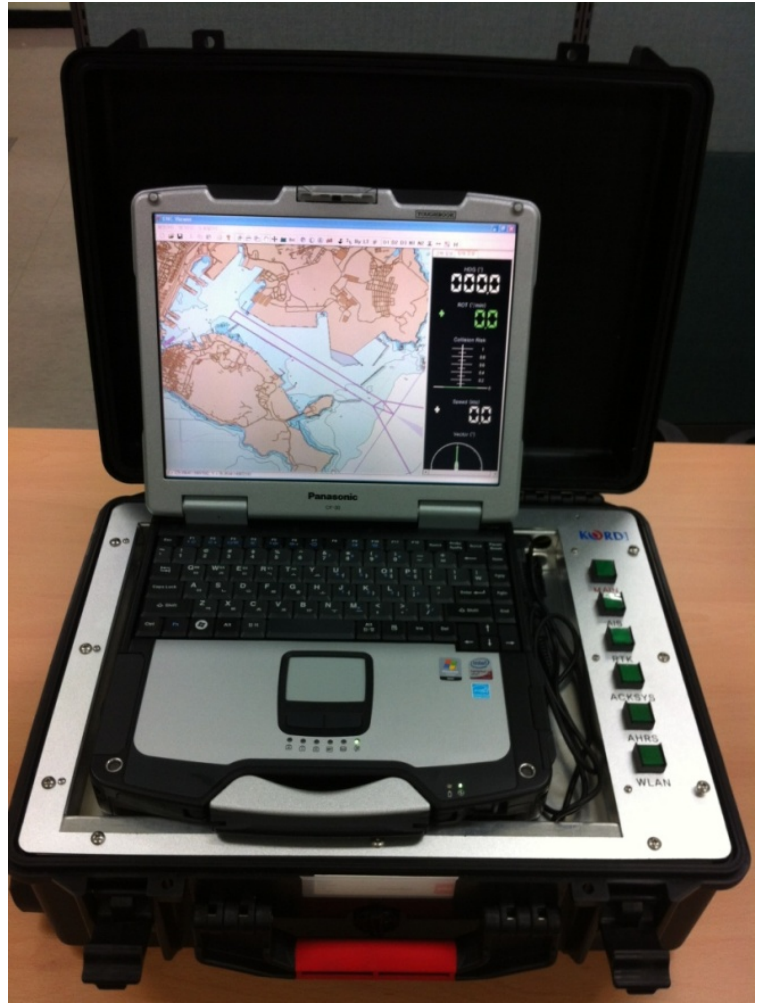

Fig. 4. Photograph of test-bed of CRMS [own photo]

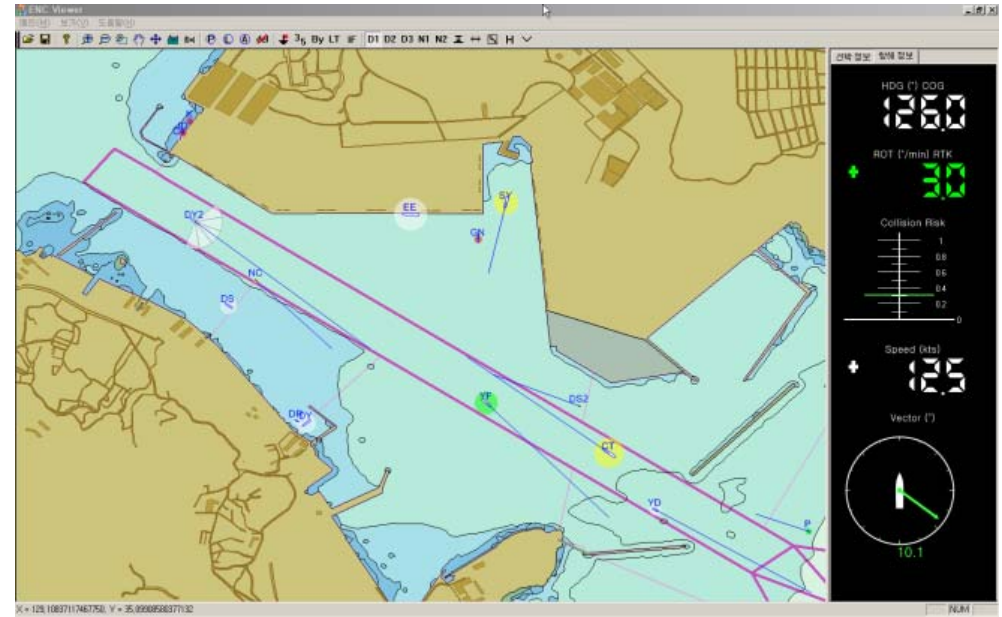

Fig. 5. Graphic user interface of CRMS [own study] 
GUI has an electronic chart based window, in which land area, buoy, fairway and ships are displayed in real time. Especially, the colors of ship and directional collision risk can be changed according to the level of collision risk. Safe ship and direction are white. Marginally safe ship and direction are green. The colors of ship and direction, which needs caution, are yellow. Dangerous ship and direction are red.

\section{SIMULATION AND RESULTS}

To test the performance of estimation algorithm of collision risk of multiple ships, AIS data of actual ship collision accident of Busan area in 2009 are collected from GICOMS (General Information Center on Maritime Safety and Security) of Korea. For replay simulation of AIS data, the simulator, from which AIS data is transmitted to CRMS in real time according to the order of log time, is generated.

\section{Simulation Test-bed based on AIS simulator}

To check into the reliability of estimation algorithm of collision risk, simplified test-bed is established by using AIS simulator as shown in fig. 6. AIS simulator generates the NMEA sentence in real time according to the traffic information in AIS log file and it transmits to CRMS through RS-232 serial communication. CRMS estimates the collision risk of multiple ships and their collision risk is displayed in ENC chart. Fig. 7 shows the GUI of AIS simulator.

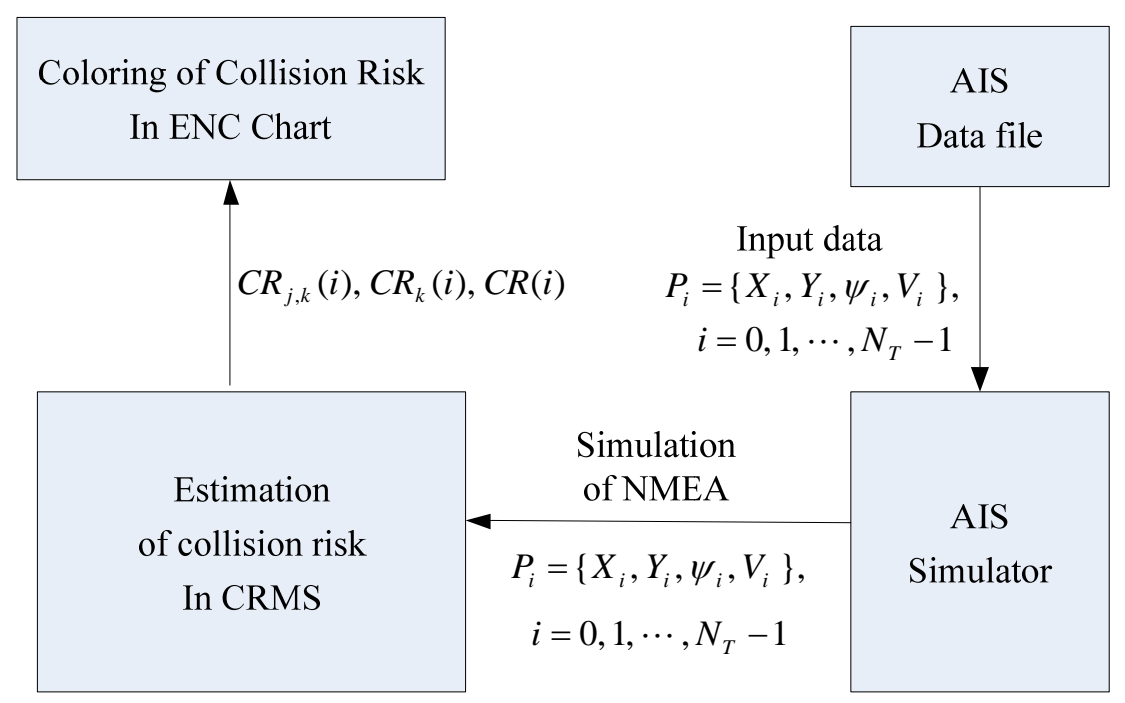

Fig. 6. Configuration of simulation test-bed based on AIS simulator [own study] 


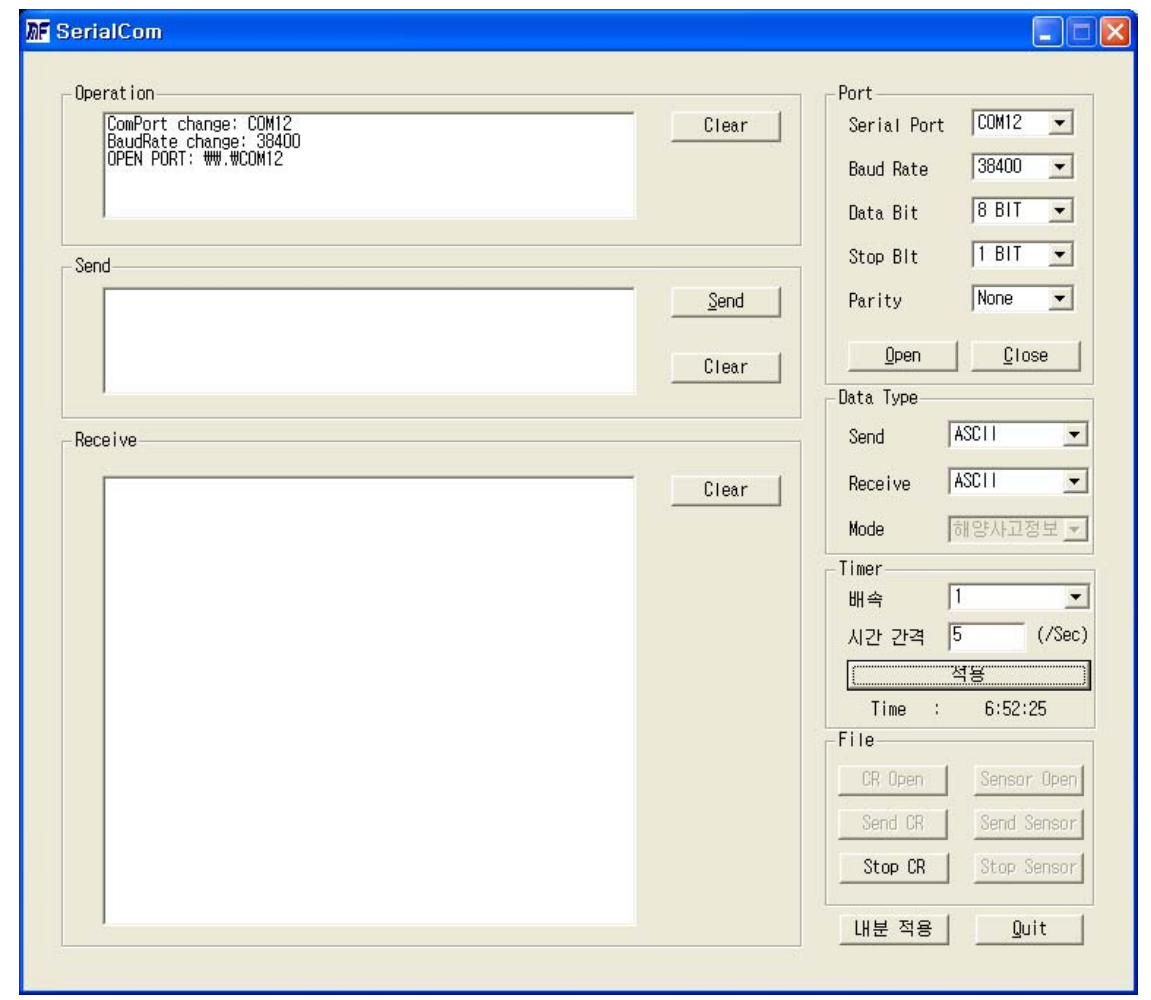

Fig. 7. Graphic user interface of AIS simulator [own study]

\section{AIS data of actual ship collision accident}

From GICOMS, AIS data of actual ship collision accidents in sea area of Busan, Korea are collected. They include 244 ships' data for about 2 hours from 05:19 to 07:19 on June 22, 2009. Out of 244 ships' data, abnormal data and data of ships, which are going far from coastal area, are excluded. So, 18 ships' data are generated in AIS simulator. Fig. 8 shows the colliding situation between product carrier, ship SY and cargo carrier, ship DY2 in Busan, Korea (KMST). Product carrier, ship SY departed from Dongmyung harbor and it was entering into departure fairway. Cargo carrier, ship DY2 departed from harbor NO. 7 and it was departing in fairway. Ship SY did not recognize ship DY2 because ship SY looked out for other arriving ship only without caution about ship DY2. Cargo carrier was speeding over 12 knots in fairway without action for collision avoidance. The first recognition between them was done at 3 minutes before collision and VHF communication was done at most 80 seconds before collision. 


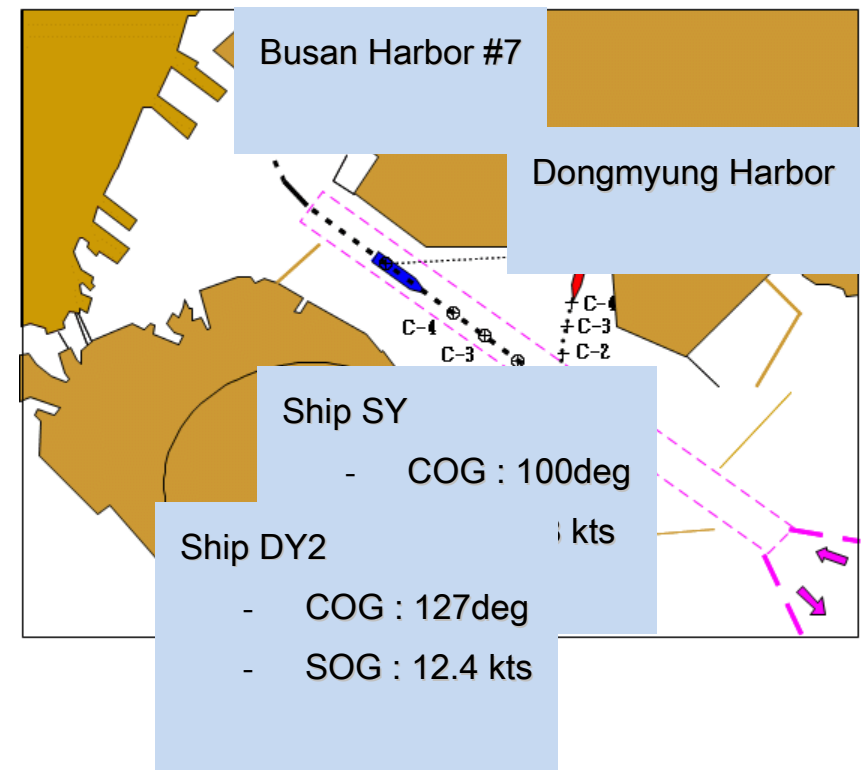

Fig. 8. Colliding situation between product carrier, ship SY and cargo carrier, ship DY2 in Busan, Korea [own study]

To test the performance of estimation of collision risk and recognition about dangerous ship in CRMS, replay simulation is carried out on the ship collision accident by using AIS simulator. Especially, it is assumed that CRMS is installed in product carrier, Ship DY2.

\section{Simulation results}

Fig. 9 to fig. 13 show examples of captured image of CRMS during replay simulation. Through replay simulation of AIS data, the variation of collision risks of ships can be found according to the change of colliding situation.

As shown in fig. 9, cargo carrier, Ship DY2 had entered into fairway and it had no collision risk in any direction at 6 minutes 18 seconds before collision. But product carrier need to be cautious because small ship in front of ship SY stopped and other big ship CT was arriving for inner harbor.

In fig. 10, the collision risk of ship DY2 for heading direction and port side increased due to arriving ship CT and product carrier ship SY, respectively. It can be found that yellow ship SY continued to need caution. But according to the written verdict of Korean Maritime Safety Tribunal (KMST), both ship DY2 and ship SY did not recognize the colliding risk between them at the 5 to 6 minutes before collision, actually. 


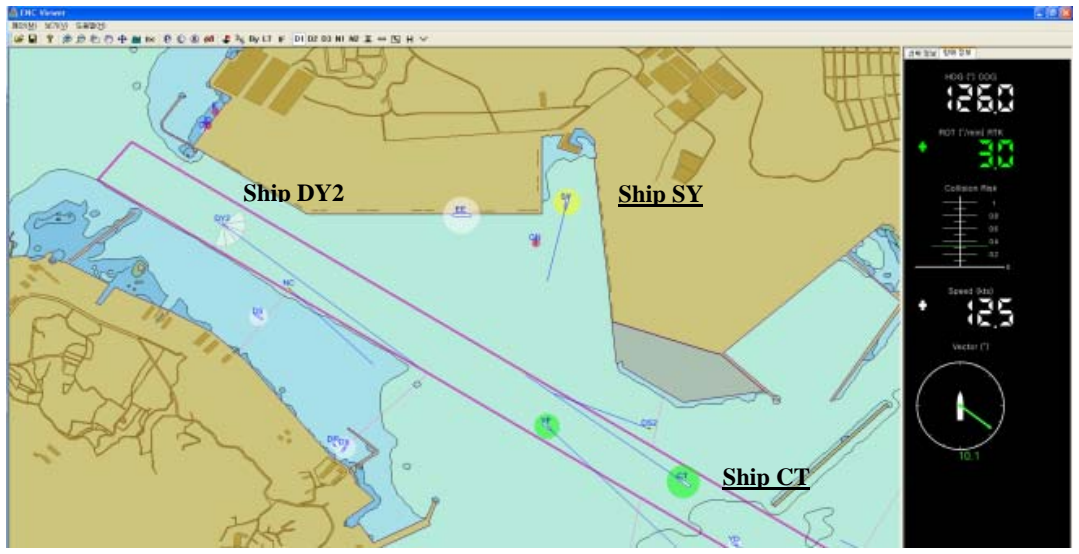

Fig. 9. Departure of product carrier, ship SY (6 min $18 \mathrm{sec}$ before collision) [own study]

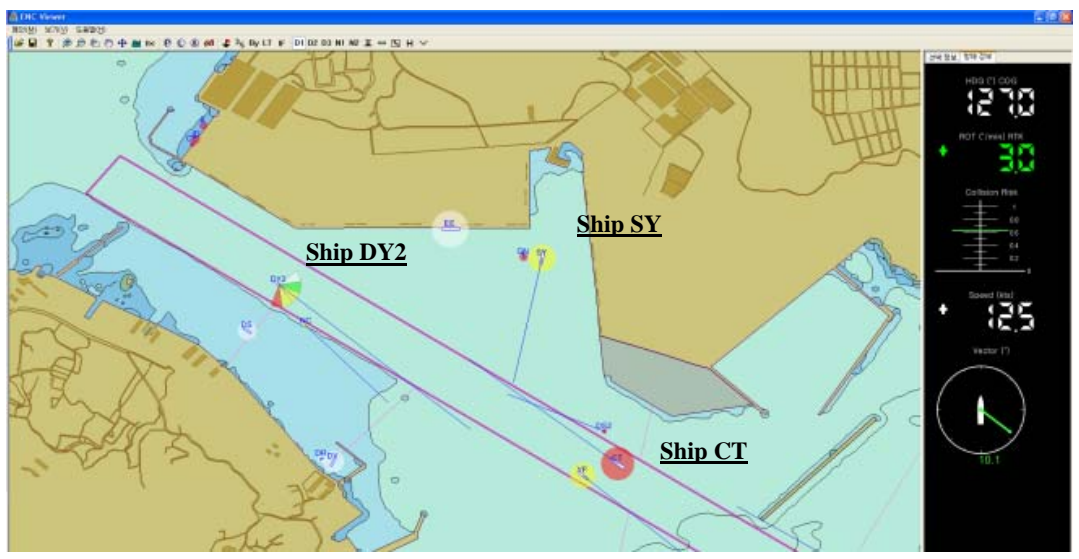

Fig. 10. Increase of collision risk due to product carrier, ship SY and other arriving ship CT (5 min before collision) [own study]

After recognition of the colliding risk of arriving ship CT, product carrier, ship SY changed its course to starboard side. So, the collision risk of ship SY decreased to be marginally safe in fig. 11 . But, expecting ship CT to pass by, ship SY tried to enter into fairway, again without considering the collision risk of ship DY2. So, as shown in fig. 12, ship CT passed by ship SY but the collision risk of ship SY did not decreased. Of course, the directional collision risk of ship DY2 in port side increased to be very dangerous. According to the written verdict of KMST, ship SY and ship DY2 recognized each other about 3 minutes before collision for the first time but the colliding situation had already become worse. 


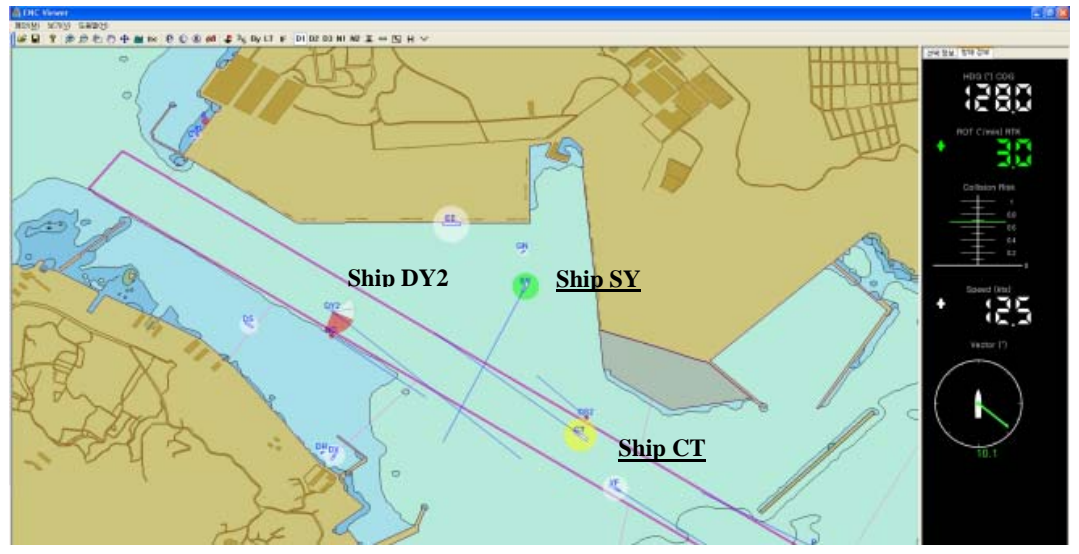

Fig. 11. Course change of product carrier, ship SY (4 min before collision) [own study]

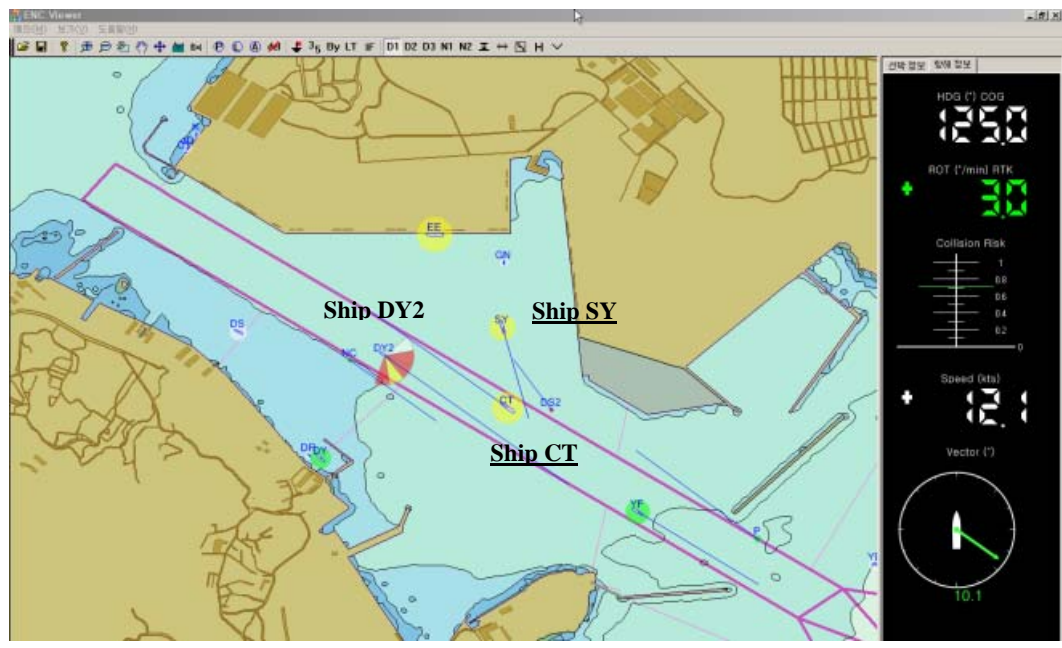

Fig. 12. Other arriving ship CT passing by product carrier, ship SY (2 min $28 \mathrm{sec}$ before collision) [own study]

As shown in fig. 13, ship SY entered into fairway with expecting ship DY2 to decrease speed but ship DY2 continued to speed over 12 knots. It can be found that the collision risk of ship SY and port side risk of ship DY2 are very dangerous value. Actually, at 80 seconds before accident, ship SY tried to communicate to ship DY2 via VHF for collision warning but it was too late. Finally, ship DY2 and ship SY collided with each other. 


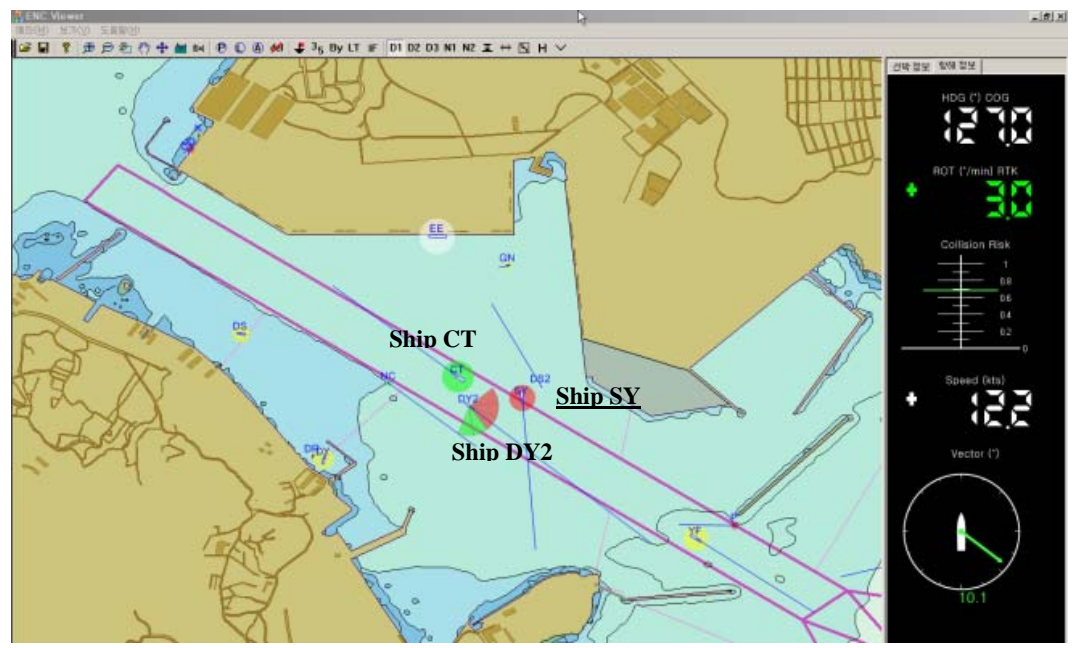

Fig. 13. Colliding situation between product carrier, ship SY and cargo carrier DY2 (58 sec before collision) [own study]

Through replay simulation of actual ship collision accident based on AIS data, newly-developed collision risk monitoring system (CRMS) displays the collision risks among ships and their changes according to the change of colliding situation reasonably. The constant value of criteria, which decides the color of ship and its level of colliding situation, should be continuously tuned through a number of validations based on actual AIS data and on-board field test.

\section{CONCLUSIONS}

To reduce crewmembers' error and help them recognize dangerous traffic ship to collide with their own-ship, a collision risk monitoring system (CRMS) is newly-developed. In CRMS, collision risks among multiple ships are estimated by using AIS data on a basis of fuzzy algorithm in real time. To test the performance of CRMS, AIS data of actual ship collision accident near sea area of Busan, Korea are collected. Through replay simulation of AIS data, it can be found that CRMS estimates collision risks of multiple ships normally according to the change of colliding and recognize dangerous direction and ship to collide with ownship.

In the future, it is necessary to adjust the criteria, which decides the level of collision risk and the colour of ship risk and directional collision risk, through a number of validations. 


\section{Acknowledgments}

This research was a part of the project titled Development of recognition system of ship collision risk (PMS2390) funded by the Ministry of Land, Transport and Maritime Affairs, Korea.

\section{REFERENCES}

[1] Gong I. Y. et al., The Development of a Supporting System for Safe Navigation on a basis of a risk, $3^{\text {rd }}$ Research Report (Development of a core technology for a risk reduction), 2002.

[2] Gong I. Y., A Review on the Characteristics of Environmental Stress Model for Maritime Traffic Safety Assessment, Journal of Korean Navigation and Port Research, 2003.

[3] Hara K. et al., A Safe Way of Collision Avoidance Maneuver based on Maneuvering Standard using Fuzzy Reasoning Model, Proceedings of MARSIM ‘93, 1993.

[4] Hasegawa K. et al., Ship Auto-navigation Fuzzy Expert System (SAFES), Journal of the Society of Naval Architecture of Japan, 1989, Vol. 166.

[5] Imazu H., Koyama T., The Determination of Collision Avoidance Action, Journal of Japan Institute of Navigation, 1984, Vol. 70.

[6] Inoue K. et al., Modeling of Mariners’ Perception of Safety when Being Faced with Imminent Danger, Journal of Japan Institute of Navigation, 1998.

[7] Kao S. L. et al., A Fuzzy Logic Method for Collision Avoidance in Vessel Traffic Service, The Journal of Navigation, 2007, Vol. 60.

[8] Kijima K., Furukawa Y., Automatic Collision Avoidance System Using the Concept of Blocking Area, Proceeding of MCMC '03, 2003.

[9] Kijima K., Furukawa Y., Design of Automatic Collision Avoidance System using Fuzzy Inference, Proceeding of CAMS ‘01, 2001.

[10] Lee H. J., Rhee K. P., Development of Collision Avoidance System by using Expert System and Search Algorithm, Journal of International Shipbuilding Progress, 2001, Vol. 48, No. 3.

[11] Son N. S., Gong I. Y., Kim S. Y., Development of Collision and Grounding Risk Monitoring System of a Ship by using Fuzzy Algorithm and Environmental Stress Model, Proceedings of MARSIM ‘06, 2006.

[12] Son N. S., Gong I. Y., KIM S. Y., Study on the Estimation of Collision Risk of Ship in Ship Handling Simulator using Fuzzy Algorithm and Environmental Stress Model. Journal of Navigation and Port Research, 2009, Vol. 33, No. 1. 
[13] Son N. S., Kim S. Y., Validation on the Algorithm of Estimation of Collision Risk among Ships based on AIS Data of Actual Ships' Collision Accident, Journal of Navigation and Port Research, 2010, Vol. 34, No. 10.

[14] Son N. S., Kim S. Y., Oh J. Y., Study on an algorithm for the estimation of collision risk among ships by using AIS database, Proceedings of ACMSSR 2009.

[15] Szlapczynski R., A Unified Measure Of Collision Risk Derived From The Concept of a Ship Domain, The Journal of Navigation, 2006, Vol. 59.

[16] The causes of maritime accidents according to the kind of accident (in Korean), http://www.kmst.go.kr/statistics/statisticsLatest.asp.

[17] Wilson P. A., Harris C. J., A Line of Sight Counteraction Navigation Algorithm for Ship Encounter Collision Avoidance, The Journal of Navigation, 2006, Vol. 56.

Received May 2012

Reviewed September 2012 